ANTHONY SAUDRAIS

ORCID: 0000-0001-9619-8135

Université Rennes 2

anthonyetyoshi@hotmail.fr

\title{
AU SERVICE DE RICHELIEU. ÉCRIRE, JOUER ET PUBLIER EUROPE
}

\section{UNE ÉCRITURE COLLABORATIVE AU SERVICE DE LA POLITIQUE DE RICHELIEU}

\subsection{DEUX AUTEURS POUR UNE PIÈCE}

Dans leur Histoire du théâtre Français, Claude et François Parfaict commencent par évoquer, dans l'examen de la comédie héroïque Europe ${ }^{1}$, la paternité d'écriture qui ne constitue à leurs yeux aucun doute : «Quoique cette pièce ait été imprimée comme d'un anonyme, cependant le nom de l'auteur était aussi connu, que l'allégorie facile à appliquer à la conjoncture des affaires, où l'Europe se trouvait alors $»^{2}$. Cet anonyme « aussi connu » n'était autre que le Cardinal de Richelieu, nommé en 1624 premier ministre de Louis $\mathrm{XIII}^{3}$. Passionné de théâtre ${ }^{4}$, protecteur de grands poètes et dramaturges comme Pierre Corneille ou Guillaume Colletet, il

1 J. Desmarets de Saint-Sorlin et Richelieu, Europe, H. Le Gras, Paris 1643.

2 C. Parfaict et F. Parfaict, Histoire du théâtre François, vol. 6, P.G. Le Mercier et Saillant, Paris 1746, p. 266.

${ }^{3}$ Sur la vision de l'État et la politique menée par Richelieu, voir F. Hildesheimer, Richelieu, une certaine idée de l'État, Publisud, Paris 1985.

${ }^{4}$ G. Couton, «Richelieu et le théâtre », [dans :] Richelieu et la culture, R. Mousnier (dir.), Éditions du CNRS, Paris 1987, pp. 79-101 ; C. Guillot, «Richelieu et le théâtre », Transversalités 1, 2011, pp. 85-102. Lire enfin M. Bayard, « Le roi au cœur du théâtre : Richelieu met en scène l'Autorité », [dans :] L'image du roi de François Ir à Louis XIV, Thomas W. Gaehtgens et N. Hochner (dir.), Maison des sciences et l'homme, Paris 2006, pp. 191-208. 
surveilla vraisemblablement la rédaction de la Déclaration du roi Louis XIII au sujet des Comédiens signée le 16 avril 1641, le document stipulant de ne plus « représenter aucunes actions malhonnêtes, ni d'user d'aucunes paroles lascives, ou à double entente, qui puisse [sic] blesser l'honnêteté publique, et sur peines d'être déclarés infâmes $[\ldots] \|^{5}$. Cette Déclaration, en accord avec le renforcement de la politique étatique menée par Richelieu, illustre la mainmise du pouvoir dans les affaires artistiques du royaume, cette nouvelle législation étant signée quelques mois avant que ne commence l'écriture d'Europe, de toute évidence entreprise au début de l'année 1642. Pourtant, la paternité d'Europe ne revient pas seulement à Richelieu mais également à son proche collaborateur, Jean Desmarets de Saint-Sorlin ${ }^{6}$, un ami du Cardinal $^{7}$. En effet, l'année précédente, sa tragi-comédie Mirame — dont on a vu quelques maximes proches de la pensée politique de Richelieu, ce qui n'exclut pas une collaboration entre les deux hommes - eut le privilège d'inaugurer le grand théâtre du Palais-Cardinal le 14 janvier $1641^{8}$. La même année, Desmarets publiait ses Euvres poétiques avec une «Épître » élogieuse dédiée au Cardinal de Richelieu. Une estampe de Pierre Lombart réalisée d'après un portrait d'Henri Gascard rappelle l'importance du poète nommé conseiller du roi, contrôleur général de l'extraordinaire des guerres ainsi que secrétaire général de la marine. Il n'y a donc rien d'étonnant que la comédie héroïque ait été écrite par ces deux hommes, Europe proposant une allégorie favorable à la politique du $\mathrm{Cardinal}^{9}$ pendant les derniers mois de ministériat, Richelieu ayant expiré le 4 décembre 1642. Sa mort fut relayée par la Gazette le 6 décembre : «Le 4 de ce mois sur le midi mourut dans son palais de cette ville en la $58^{\mathrm{e}}$ année de son âge le Cardinal Duc de Richelieu premier ministre d'État de notre invincible monarque : laquelle charge il a exercé depuis 20 ans dignement, qu'il n'y a point de paroles assez relevées pour le bien exprimer $[\ldots] »^{10}$. Europe, qui s'inscrit dans la lignée des pièces allégoriques et politiques d'actualités antérieures ${ }^{11}$, représente sur le plan théâtral le testament politique de Richelieu.

${ }^{5}$ C. Parfaict et F. Parfaict, op. cit., vol. 6, p. 132.

${ }^{6}$ Roland Mousnier attribue l'écriture de cette pièce à Desmarets dans son ouvrage L'homme rouge ou la vie du cardinal de Richelieu, R. Laffont, Paris 1992, p. 652.

7 Son théâtre - Aspasie (1636), Les Visionnaires (1637), Scipion (1638), Roxane (1640), Mirame (1641), Erigone (1642) et Europe (1642) — a bénéficié d'une édition complète, Théâtre complet de Desmarets (1636-1643), textes établis, présentés et annotés par C. Chaîneaux avec un dossier iconographique de C. Guillot, coll. « Sources classiques », Honoré Champion, Paris 2005.

${ }^{8}$ Le spectacle bénéficia de belles estampes de Stefano della Bella. Elles sont commentées par Catherine Guillot dans son article « Les illustrations de Mirame de Desmarets par S. Della Bella », Revue d'Histoire du Théâtre 218, 2003, pp. 145-160. Le livre de fête a quant à lui été l'objet d'une étude de la part de Benoît Bolduc dans son article "Mirame, fête théâtrale dans un fauteuil ? ", Revue d'Histoire du Théâtre 245-246, 2010, pp. 159-172.

${ }^{9}$ G. Hall, «Europe, allégorie théâtrale et propagande politique », [dans :] L'âge d'or du mécénat, Colloque du CNRS, Paris 1985, pp. 319-327.

10 La Gazette, 1642, p. 1155.

11 On pense notamment aux pièces politiques et allégoriques écrites pendant les guerres de religion. Sur le sujet, voir Ch. Bouteille-Meister, « De l'appel à la révolte protestante à la sortie 


\subsection{L'HISTOIRE ET LE CONTEXTE POLITIQUE. LA GUERRE FRANCO-ESPAGNOLE}

L'action de la pièce met en scène l'affrontement entre la France, représentée par Francion, et l'Espagne représentée par Ibère. Au prologue, la France affirme son vœu de conserver la liberté d'Europe importunée par la soumission que souhaite lui imposer Ibère, la Paix descendant du ciel pour tenter de résoudre le conflit :

\section{La Paix, descendant du Ciel}

D'un tranquille séjour de la voûte azurée, Je reviens aux climats des mortels habités, Pour combler de félicités

Ces lieux où je suis désirée ${ }^{12}$.

Durant les cinq actes, la pièce met en scène les diverses machinations menées par Ibère soutenu par ses alliés européens, la perfidie de l'Espagne trouvant de l'appui chez Germanique comme chez la nymphe Ausonie, représentant l'Italie, Germanique agrandissant au fil de l'intrigue ses soutiens politiques en convoquant les services de Parthénope et de Mélanie qui représentent Naples et Milan. Mais Ibère échoue à conquérir Europe, cette dernière achevant la pièce sur ces vers de réconciliation entre Francion et Germanique :

\section{Europe}

Tous deux étant d'accord

Vous me donnez la paix : je ne crains nul effort.

Que le Ciel, Francion, toujours vous favorise,

Et vos chers alliés auteurs de ma franchise.

Germanique en mon cœur tiendra à ce même rang.

Je vous aimerai tous : vous êtes tous mon sang.

Ibère l'est aussi : s'il étouffe sa flamme

Je lui réserve encore une place en mon âme ${ }^{13}$.

La pièce proposait une apologie à la gloire de la politique de Richelieu qui avait commencé la guerre contre l'Espagne en 1635. Le Cardinal s'était engagé dans la Guerre de Trente Ans qui s'acheva, quelques années après sa mort, par la signature du traité de Westphalie le 24 octobre 1648. Pourtant, la guerre contre l'Espagne ne se termina qu'en 1659 avec la signature du Traité des Pyrénées scellant le mariage entre Louis XIV et l'infante d'Espagne Marie-Thérèse d'Autriche. Ainsi, Europe

de crise catholique : les usages de la violence sur les corps dans le théâtre d'actualité en français pendant les guerres de religion », Littératures classiques 3, vol. 73, 2010, pp. 265-272. Par exemple, Cléophon, une pièce de Jacques de Fonteny publiée en 1600, met en scène la mort d'Henri III. Elle a été publiée dans une édition moderne, Théâtre de la cruauté et récits sanglants en France (XVI $X V I I^{e}$ s.), Chr. Biet (dir.), coll. « Bouquins », Robert Laffont, Paris 2006.

12 J. Desmarets de Saint-Sorlin et Richelieu, op. cit.

13 Ibidem. 
proposait la synthèse des différents conflits européens que le Cardinal laissait à ses successeurs, en particulier son protégé et futur régent, le Cardinal Mazarin. Pour défendre Richelieu, Francion formule des arguments à l'encontre de la politique dominatrice d'Ibère comme son occupation tyrannique de l'Amérique, Europe lui demandant : « Prétendez-vous quitter Amérique ma sœur? » (I, 5). Et l'Espagne de lui répondre : " Non, car contre vous-même elle m'est secourable ». De facto, ces vers reprochent à l'Espagne sa politique de colonisation poursuivie par Philippe IV, en l'occurrence l'entreprise de la Nouvelle-Espagne ${ }^{14}$ alors que la fin de l'union Ibérique venait d'être scellée depuis 1640, notamment par la déclaration d'indépendance du Portuga $1^{15}$. Avec le personnage d'Ibère, l'Espagne est montrée comme un monstre sanguinaire, son entreprise coloniale lui permettant de conquérir le cœur d'Europe : « Son sang m'est un moyen pour gagner votre cœur ». La France incarne, par l'intermédiaire de Francion, cette position nuancée nécessaire à la résolution des conflits européens contre la passion, néfaste à l'obtention de la paix, de son principal ennemi espagnol. Plus intimement, Richelieu écrivait dans une lettre datée du 3 janvier 1630, adressée au duc de Créquy, que «L'Espagne ne fait jamais rien volontairement qu'en sa faveur, et la force est le seul motif qui la porte à faire raison à autrui ${ }^{16}$. Écriture épistolaire et théâtre se faisaient donc écho.

\section{LES PROBLÈMES DE LA REPRÉSENTATION}

\subsection{QUEL PUBLIC POUR QUELLE SALLE?}

D'emblée, la question de la représentation se pose : Europe a-t-elle été jouée du vivant de Richelieu ? Si oui, pour quel public ? Certains spécialistes estiment que le Cardinal assista à une représentation de la comédie le 18 novembre $1642^{17}$. Pourtant, la mode était portée à l'époque sur le genre de la tragi-comédie par des auteurs comme Scudéry, Boisrobert, Colletet, Desfontaines, Sallebray ou Gillet de la Tessonnerie ${ }^{18}$. En tenant compte de cette réalité, Europe apparaît comme une exception dans le paysage théâtral parisien. Son écriture singulière, voire une certaine opacité dans la compréhension du canevas dramatique, ne favorisaient pas une

14 Pour une synthèse de l'Amérique ibérique, voir T. Calvo, L'Amérique ibérique de 1570 à 1910, Nathan, Paris 1994 ; sur l'histoire de la colonisation européenne et ses différents impacts en Amérique, voir P. Chaunu, L'expansion européenne du XIII siècle au XV siècle, Presses universitaires de France, Paris 1969.

15 Sur l'histoire et l'indépendance du Portugal, voir A. Henrique de Oliveira Marques, Histoire du Portugal et de son empire colonial, coll. « Hommes et sociétés », Karthala, Paris 1998.

16 Richelieu, Maximes d'État et fragments politiques du Cardinal de Richelieu, publiés par M.G. Hanotaux, Imprimerie nationale, Paris 1880, p. 25.

17 J. Wollenberg, «Richelieu et le système européen de sécurité collective. La bibliothèque du Cardinal comme centre intellectuel de la vie politique », Dix-septième siècle 210, 2001, p. 99.

18 Sur la poétique de la tragi-comédie, voir H. Baby, La tragi-comédie de Corneille à Quinault, Klincksieck, Paris 2001. 
représentation parisienne, Paris comptant à l'époque trois grands théâtres. Le premier était l'Hôtel de Bourgogne, le plus ancien de la capitale ${ }^{19}$. Sa scénographie usait de décors compartimentés ${ }^{20}$ conçus par le décorateur et machiniste Georges Buffequin qui expire, la même année que Mirame, en $1641^{21}$. La deuxième grande salle était le théâtre du Marais, situé rue Vieille-du-Temple ${ }^{22}$. Corneille y représenta Médée et la retentissante tragi-comédie du Cid. Mais la situation financière du Marais n'était pas toujours stable et moins pérenne que celle de l'Hôtel de Bourgogne, la troupe étant régulièrement en déplacement dans le royaume de France pour jouer son répertoire. Une représentation d'Europe au Marais semble aussi délicate à prouver qu'une représentation à l'Hôtel de Bourgogne même si Richelieu préférait la troupe de la rue Vieille-du-Temple à celle de la rue Mauconseil. Enfin, la troisième hypothèse serait une représentation d'Europe dans le nouveau théâtre du Palais-Royal, futur théâtre de Molière et de l'Académie royale de musique, inauguré le 14 janvier 1641 avec Mirame. Techniquement, la scène était propice à la représentation de la comédie héroïque, notamment pour manœuvrer depuis les cintres la descente de la Paix au prologue, la grande salle du Palais-Royal étant équipée de la plus puissante et moderne machinerie théâtrale de France avant celle installée par Torelli au Petit-Bourbon en 1645 pour la représentation de La Finta Pazza. Pourtant tout, dans l'écriture de la pièce, nous amène à penser qu'une représentation d'Europe se soit produite dans l'intimité du Cardinal et non dans une grande salle de théâtre parisienne.

\subsection{L'HYPOTHÈSE DU PETIT THÉÂTRE DE RICHELIEU AU PALAIS-CARDINAL}

En effet, pour combler sa passion du théâtre, Richelieu aménagea une petite salle de spectacle dans l'une des ailes du Palais-Cardinal. Elle se situait dans l'actuelle Galerie des Proues. Elle est mentionnée par Henri Sauval :

Bien davantage, comme si ce n'eut pas été assez d'un théâtre dans son palais, il lui en fallut deux,

19 Sur l'histoire de l'Hôtel de Bourgogne, voir S.W. Deierkauf-Holsboer, Le théâtre de l'Hôtel de Bourgogne, Nizet, Paris 1968-1970, 2 vol. Sur l'évolution architecturale de ce théâtre, voir P. Pasquier, "L'Hôtel de Bourgogne et son évolution architecturale : éléments pour une synthèse », [dans :] Les lieux du spectacle dans l'Europe du XVII siècle, Actes du colloque du Centre de recherches sur le XVII ${ }^{\mathrm{e}}$ siècle européen, Université Michel de Montaigne - Bordeaux III, 11-13 mars 2004, Ch. Mazouer (dir.), Narr Verlag, Biblio 17, Tübingen 2006, pp. 47-71.

${ }^{20}$ Sur la scénographie de ces décors compartimentés, voir A. Surgers, " "Ils ont dû faire choix". Quelques pistes pour une lecture et une interprétation des décors à compartiments », [dans :] La scène et la coulisse dans le théâtre du XVII siècle en France, Actes du colloque de la Sorbonne, Paris, janvier 2006, G. Forestier et L. Michel (dir.), coll. « Theatrum mundi », Presses de l'Université Paris-Sorbonne, Paris 2011, pp. 295-306.

21 Sur le machiniste, voir $\mathrm{H}$. Visentin, « Décorateur à la cour et à la ville : un artisan de scène nommé Georges Buffequin (1585 ? - 1641), XVII siècle 195, 1997, pp. 325-339 ; M. Bayard, « Les faiseurs d'artifices : Georges Buffequin et les artistes de l'éphémère à l'époque de Richelieu ", XVII siècle 230, 2006, pp. 151-164.

22 Sur l'histoire du théâtre du Marais, voir S.W. Deierkauf-Holsboer, Le Théâtre du Marais, Nizet, Paris 1954-1958, 2 vol. 
un petit et un grand ; l'un capable de contenir six cent personnes, et l'autre plus de trois mille. Dans le petit, il assistait aux pièces de théâtre que les comédiens représentaient ordinairement au Marais du temple : le grand était réservé pour les comédies de pompe et de parade, quand la profondeur des perspectives, la variété des décorations, la magnificence des machines y attiraient leurs majestés et la $\operatorname{cour}^{23}$.

Europe n'avait aucun besoin d'être représentée au Marais puisque la troupe venait régulièrement jouer dans ce petit théâtre confidentiel. Un plan du rez-dechaussée du Palais-Cardinal daté de 1633 par Alexandre Gady ${ }^{24}$ (ce plan ne mentionne pas encore le théâtre de Mirame) schématise une pièce rectangulaire abritant un théâtre. Pour Philippe Cornuaille, le griffonnage n'est pas d'époque : il s'agit selon lui d'une annotation postérieure ${ }^{25}$. De quelle époque date sa construction? Victor Champier mentionne un contrat passé avec Georges Buffequin pour la construction d'un théâtre dans l'hôtel de Richelieu en 1635, le machiniste ayant reçu pour cette tâche une rémunération de 300 livres pour avoir travaillé aux décorations $^{26}$. Inaugurée en 1635 , la scène allait accueillir quelques créations dramatiques parmi lesquelles La Comédie des Tuileries écrite par les cinq auteurs protégés du Cardinal : François de Boisrobert, François Colletet, Pierre Corneille, Jean de Rotrou et Claude de l'Estoile. Sur le plan scénographique, la scène permettait de réaliser quelques changements de décors ${ }^{27}$. Dans ce lieu propice à l'intimité, Richelieu fit représenter de nombreuses pièces, parfois confidentielles, comme le laisse sous-entendre Henriette de La Tour d'Auvergne affirmant, dans une lettre du 12 décembre 1639, que « Monsieur le Cardinal arriva hier au soir en cette ville [Paris], d'où il doit partir demain. Il fait jouer aujourd'hui une comédie chez lui qui est, dit-on, le sujet de son voyage ici $»^{28}$. Or nous savons que ce théâtre fut préservé, du moins partiellement, après la mort de Richelieu. En effet le machiniste de Louis XIV, Carlo Vigarani ${ }^{29}$, l'aménagea pour accueillir

${ }^{23}$ H. Sauval, Histoire et recherches des antiquités de la ville de Paris, vol. 2, Moette, Paris 1724, p. 161.

24 A. Gady, Jacques Lemercier, architecte et ingénieur du Roi, Éditions de la Maison des sciences de l'homme, Paris 2005, p. 297.

25 P. Cornuaille, Les décors de Molière (1658-1674), Presses de l’Université Paris-Sorbonne, Paris 2015, p. 71.

26 V. Champier et G.-R. Sandoz, Le Palais-Royal d'après des documents inédits (1629-1900), vol. 1, Société de propagation des livres d'art, Paris 1900, p. 29.

27 La préface de la pièce commence par cette remarque : « Cette pièce, lecteur, a été trop bien concertée, pour n'être pas dans la justesse requise, et pour ne point contenter vos yeux, après avoir charmé vos oreilles. Vous savez avec quelle magnificence, elle a été représentée à la Cour, et que ceux qui l'ont vue en ont tous admiré la conduite, et les décorations du théâtre » ("Au lecteur », [dans :] La Comédie des Tuileries, A. Courbé, Paris 1638).

28 Correspondance du marquis et de la marquise de La Moussaye (1619-1663), présentée par J.L. Tulot, Champion, Paris 1999, p. 118.

29 Sur le machiniste, voir J. de La Gorce, Carlo Vigarani, intendant des plaisirs de Louis XIV, coll. « Les métiers de Versailles », Perrin/Château de Versailles, Paris 2005. 
les ballets dansés par le roi entre 1663 et $1665^{30}$. Un plan de 1673 nous renseigne sur l'aménagement de l'ancien petit théâtre de Richelieu préservé à l'époque de Vigarani, la salle rectangulaire mesurant 4,7 toises de large sur 9,8 toises de long, soit 9,15 mètres sur 19,1 mètres. Le plan daté de 1633 confirme cette dimension. Sur celui de 1673, une ligne délimite la scène de la salle. Or, la comparaison entre les deux plans semble nous indiquer que la scène représentait un peu plus du tiers de l'espace contre un peu moins de deux tiers pour la salle. La scène mesurait donc approximativement 3,2 toises de profondeur, soit 6,23 mètres, pour une largeur de 9,15 mètres. Cette proportion permettait de profiter d'une scène aux dimensions limitées mais raisonnables ; le public, certes réduit, pouvait admirer avec une certaine tranquillité les décors et le mouvement des machines. Sans nul doute, ce petit théâtre était le plus propice à la représentation d'Europe.

\section{PUBLIER EUROPE}

\subsection{L'ÉDITION D'HENRY LE GRAS (1643)}

$\mathrm{Au}$ XVII ${ }^{\mathrm{e}}$ siècle, nous comptons plusieurs éditions de la pièce avec, d'une part, l'édition originale d'Henry Le Gras publiée en $1643^{31}$, enrichie d'un frontispice. Nous procéderons à une comparaison avec une réédition de la pièce, beaucoup plus modeste sur le plan éditorial, et vendue à Paris par Charles de Sercy en $1661^{32}$. Il s'agit d'une contrefaçon bruxelloise ${ }^{33}$. L'édition originale fut ainsi confiée à Henry Le Gras, marchand libraire domicilié à Paris. L'achevé d'imprimer, d'après le privilège, est daté du 13 janvier 1643. Elle est de bonne qualité, notamment dans le papier utilisé ; son format est agréable et la typographie soignée. À l'ouverture du livre, nous découvrons un frontispice daté de 1643. Malheureusement, il ne comporte pas le nom du dessinateur ni du graveur. L'image représente six personnages dont cinq sont disposés sur une ligne isocéphale avec, de gauche à droite, Francion reconnaissable par le personnage masculin qui emprunte ses traits au visage de Louis XIII ; un coq vient se poser sur le casque militaire de la France prête à dégainer son épée de la main droite pour défendre, sur sa gauche, la reine Europe dont le visage exprime l'effroi, un ressentiment accentué par le mouvement élancé de ses mains rejetant l'esclavage imposé par l'Espagne. La représentation d'Europe n'est pas sans rappeler la figure de la Vierge par le

30 Sur l'histoire et le réaménagement de ce petit théâtre de Richelieu, voir A. Saudrais, Spectacles et machines au temps de Louis XIV (1659-1715), [thèse de doctorat], Université Rennes 2, pp. 39-51.

31 L'exemplaire consulté se trouve à la Bibliothèque de l'Arsenal, cote GD-41769.

32 L'exemplaire consulté se trouve à la Bibliothèque de l'Arsenal, cote GD-10016.

33 Cette information est reprise de la base internet réalisée par Alain Riffaud : « Répertoire du théâtre français imprimé au XVII ${ }^{\mathrm{e}}$ siècle » (lien internet : <http://www.unifr.ch/repertoiretheatre17/> [consulté le 29.03.2019]). 
majestueux drapé qui l'enveloppe, son air de douceur et sa couronne où culmine une croix. Ibère est représenté quant à lui sous les traits stéréotypés d'un espagnol portant une fraise volontairement démesurée, une mode encore persistante sous le règne de Philippe $\mathrm{IV}^{34}$. Pour expliciter la violence de sa passion, il tient de la main gauche une chaîne pour soumettre Europe, Ibère étant soutenu par d'autres personnages comme cet homme barbu, légèrement en retrait et tenant de la main droite la chaîne tenue par la main gauche de l'Espagne. Il s'agit de Germanique, reconnaissable par les deux aigles formant le chapeau allégorique de l'Allemagne. Une femme - la seule à ne pas être disposée sur la ligne isocéphale — pose une main sur la cuisse gauche d'Ibère et, posant l'index de sa main droite sur sa bouche, représente le secret, c'est-à-dire la machination qui se prépare contre La France et Europe. Il s'agit de l'Italie. Enfin, le dernier personnage situé au centre de la composition est une femme. En retrait, placée derrière Europe entre les deux camps qui s'affrontent, elle regarde en direction du lecteur et porte, sur sa tête, deux clés qui s'entrecroisent : elle symbolise, par allégorie, la solution. S'agit-il de la Paix qui ouvre le prologue ? Le fait que la lumière vienne se poser sur elle nous amène à poser cette hypothèse. Ensuite, une fois la page de titre tournée, le lecteur découvre un quatrain composé d'alexandrins annonçant la portée politique de la pièce :

Quiconque aime la France, aimera cet ouvrage ;

Et qui ne l'aime pas, en maudira l'auteur.

Tu me vas contenter, qui que tu sois, lecteur.

Des uns j'aime la joie, et des autres la rage ${ }^{35}$.

Aussi, « le libraire au lecteur » vient expliciter l'allégorie politique de la pièce en faveur de la politique menée par Richelieu, la pièce dénonçant « l'ambition des Espagnols pour se rendre maitres de l'Europe, et la protection que lui donne le roi avec ses alliés pour le garantir de sa servitude ». Plus rare encore dans l'impression d'une pièce de théâtre à cette époque est le fait que l'ouvrage mentionne, à la toute fin, les différentes « clés » d'interprétation pour comprendre les personnages de la pièce, certains propos tenus durant la pièce étant traduits, comme « la roche rebelle » désignant la ville de la Rochelle ou les « destructeurs d'autels » évoquant les luthériens et les calvinistes.

\subsection{L’ÉDITION DE CHARLES DE SERCY (1661)}

Enfin, il est intéressant d'évoquer ce phénomène quelque peu étrange d'une réédition de la pièce en 1661, Europe n'ayant vraisemblablement jamais été reprise, si tant soit peu qu'elle fut jouée du vivant de Richelieu. À bien des égards, cette contrefaçon bruxelloise est surprenante. En effet, pourquoi réimprimer une

34 Nombre de tableaux peints par Diego Velasquez témoignent de cette mode.

35 J. Desmarets de Saint-Sorlin et Richelieu, op. cit. 
pièce allégorique flattant l'ancienne politique de Richelieu ? La date de publication nous amène pourtant à poser une coïncidence, l'année 1661 marquant la prise de pouvoir personnel par Louis XIV après la mort de Mazarin le 9 mars. Cette publication voulait-elle faire écho au renforcement d'une politique étatique menée par Richelieu, poursuivie par Mazarin et désormais sous l'autorité absolue de Louis XIV ? Enfin, au-delà de cette coïncidence, se pose l'hypothèse d'une éventuelle reprise de la pièce à l'Hôtel de Bourgogne, le théâtre du Marais s'étant depuis le milieu des années 1640 spécialisé dans le répertoire à machines ${ }^{36}$. De surcroît, le Marais s'apprêtait à reprendre au début de l'année 1661 la tragédie à machines de Corneille et Sourdéac représentée l'hiver dernier dans le vieux château du Neubourg : La Toison d'or.

L'imprimé de Charles de Sercy, par rapport à l'édition originale d'Henry Le Gras, est de médiocre qualité. Elle comporte des différences par rapport à l'imprimé de 1643. D'abord, il n'y plus de frontispice. Le quatrain nationaliste et le « libraire au lecteur » ont disparu. Enfin, le prologue a été supprimé. En revanche, la fin de l'imprimé conserve ces fameuses clés permettant de comprendre les personnages et certaines expressions tenues par les principaux protagonistes. Bien que mystérieuse, cette réédition nous amène à constater que la comédie héroïque ne fut pas immédiatement tombée dans l'oubli, voire qu'une réactualisation de la pièce fut possible par l'une des principales troupes parisiennes, l'époque étant propice à flatter la monarchie qui, en ce début de l'année 1661, annonçait la prise de pouvoir personnelle de Louis XIV. En ce sens, réimprimer une pièce allégorique favorable à la politique de Richelieu n'avait rien d'injurieux, Louis XIV héritant d'un trône largement consolidé par l'ancien ministre de Louis XIII et renforcé, après la victoire de la Fronde, par Mazarin. Mais la France s'étant depuis peu réconciliée avec l'Espagne, notamment par la signature du Traité des Pyrénées le 7 novembre 1659, quel pouvait être l'intérêt de republier une pièce évoquant une guerre terminée entre les deux plus grandes puissances catholiques d'Europe ? Peut-être en souvenir d'une paix souhaitée dans cette pièce écrite en 1642 et qui venait, effectivement, d'être réalisée par le mariage de Louis XIV et de l'infante d'Espagne en 1659. Or une pièce allégorique et politique de Philippe Quinault, Lysis et Hespérie, représentée le 26 novembre 1660, avait célébré ce mariage ${ }^{37}$. Chappuzeau mentionne « une pastorale allégorique sur le sujet de la négociation de la paix et du mariage du roi. Elle fut composée de concert avec Monsieur de Lionne sur les mémoires qu'en fournit le Cardinal Mazarin, et représentée au Louvre par la troupe royale.

36 Sur l'évolution des répertoires et des deux principaux théâtres de Paris au milieu des années 1640, voir S. Blondet, "Les Travaux et les Jours. Réfection et création des scènes parisiennes (1644-1647) », [dans :] La scène et la coulisse dans le théâtre du XVII siècle en France, Actes du colloque de la Sorbonne, Paris, janvier 2006, G. Forestier et L. Michel (dir.), coll. « Theatrum mundi », Presses de l'Université Paris-Sorbonne, Paris 2011, pp. 57-70.

37 Le prologue de La Toison d'or de Corneille célébrait également le mariage entre Louis XIV et l'infante d'Espagne. 
Mais elle n'a pas été imprimée pour de certaines raisons, et l'original apostillé de Monsieur de Lionne est dans la bibliothèque de Monsieur Colbert $»^{38}$. Comme un écho à la comédie héroïque de Richelieu, cette nouvelle pièce mystérieuse de Quinault, futur poète de l'Académie royale de musique, poursuivait le théâtre allégorique et politique que le public retrouvera, quelques années plus tard, dans les futurs prologues de la tragédie en musique de Lully.

\section{AT RICHELIEU'S SERVICE: \\ WRITING, PLAYING, AND PUBLISHING EUROPE}

Summary

The aim of the present article is to come back to the collaborative writing, between Richelieu and Desmarets de Saint-Sorlin, of Europe, a heroic comedy praising the Cardinal's politics. We also question the representation of the play as well as the editorial policy of the original edition of 1643 and its reprint from 1661.

Key words: comedy, theatre, Richelieu, $17^{\text {th }}$-century France, Desmarets de Saint-Sorlin, book.

38 S. Chappuzeau, Le théâtre français, M. Mayer, Paris 1674, p. 107. 